\title{
INTERPRETACIONES DE LA EDUCACIÓN
} PARA LA CIUDADANÍA GLOBAL EN LA REFORMA DE LA EDUCACIÓN MEDIA SUPERIOR EN MÉXICO

\author{
Interpretations of Education for Global Citizenship in \\ the Mexican Reform of the Upper Secondary Education
}

Cecilia Peraza Sanginés *

\section{$\underline{\text { RESUMEN }}$}

En el planteamiento del monográfico, se toma como punto de partida la intensificación de las globalizaciones y la manera en que ésta ha cambiado los modelos tradicionales de la educación para la ciudadanía. Este trabajo revisa el caso mexicano a partir de la reforma integral a la educación media superior (2009), en la que se crea un marco curricular común para el bachillerato. Con dicha base, se identifican las categorías de la educación para la ciudadanía en el currículum y en el enfoque por competencias, a través del análisis del discurso pedagógico oficial, la revisión curricular y la reflexión sobre la propia práctica docente. La discusión gira en torno a la brecha que se genera entre un discurso pedagógico oficial progresista y un sistema educativo conservador, para identificar las consecuencias que tiene en la interpretación de la educación para la ciudadanía global..

${ }^{*}$ Universidad Nacional Autónoma de México (México). 
PALABRAS CLAVE: Educación para la Ciudadanía, Educación Obligatoria, Política Educativa, Prácticas Pedagógicas, Currículum Oculto, Rol de la Escuela.

\section{ABSTRACT}

In the monographic approach, it takes as its starting point the intensification of globalizations and how it has changed the traditional models of education for citizenship. This paper reviews the Mexican case from the comprehensive reform of upper secondary education (2009), in which a common curriculum framework is created. With this base, the categories of citizenship in education are identified, in the curriculum and the skills approach, through the analysis of the official pedagogical discourse, the curricular revision and the reflection on the own teaching practice. The discussion revolves around the gap between an official progressive pedagogical discourse and a conservative educational system to identify the consequences it has on the interpretation of education for global citizenship.

KEY WORDS: Citizenship Education, Compulsory Education, Politics of Education, Educational Practices, Hidden Curriculum, School Role.

$* * * * * *$

\section{INTRODUCCIÓN}

La noción de 'ciudadanía global' ha cobrado un lugar central en el discurso internacional acerca de las prioridades educativas para el desarrollo. A partir de la iniciativa mundial "La educación ante todo" (GEFI, por sus siglas en inglés) del Secretario General de las Naciones Unidas, puesta en marcha en septiembre de 2012, el 'fomento de la ciudadanía global' ha pasado a ser considerada una prioridad. No obstante, se trata de un concepto poco claro, sujeto a múltiples interpretaciones.

En este artículo se explora la manera en que las tendencias educativas globales han ido modificando el modelo tradicional de la educación para la ciudadanía en México, a partir de la reforma integral a la educación media superior, que culminó en el año 2009, con la creación del Sistema Nacional de Bachillerato con un marco curricular común (MCC).

El método utilizado para identificar las interpretaciones de la educación para la ciudadanía global en México es, por un lado, el análisis del discurso, con base en los documentos oficiales de la política educativa mexicana, como son el documento de Reforma Integral a la Educación Media Superior (SEMS, 2008) y el Plan Sectorial de Educación 2013 - 2018, para identificar los elementos de recontextualización del discurso internacional en el campo oficial. Por otro lado, se hace una revisión curricular con base en 
libros de texto de bachillerato y una reflexión desde la propia práctica docente.

La discusión está inspirada en el modelo analítico de BERNSTEIN (1990) sobre el dispositivo pedagógico de reproducción que va desde la identificación del discurso hegemónico en el campo internacional hasta su recontextualización en el campo pedagógico.

Las reflexiones que se exponen en este artículo, resultan de un trabajo de investigación más amplio (PERAZA, 2015), desarrollado entre agosto de 2014 y noviembre de 2015, que explora los procesos de recontextualización del discurso oficial sobre educación para la ciudadanía global en el campo pedagógico, a partir del trabajo etnográfico y la observación participante en un colegio de bachillerato general con enfoque propedéutico en Nayarit, México.

La tesis central es que existe una brecha entre el discurso oficial mexicano -que recontextualiza planteamientos internacionales progresistas- y la operacionalización de la educación para la ciudadanía, en un sistema educativo conservador. Esa brecha se intensifica con las constantes reformas educativas que se han suscitado en los últimos años y obstaculiza la transformación del modelo tradicional de la educación para la ciudadanía, por el escepticismo con que es recibido por los actores, especialmente el personal docente.

Para fundamentar este trabajo, se recuperan algunas reflexiones teóricas sobre el concepto de ciudadanía global en las políticas educativas. Las interpretaciones que se hacen de la ciudadanía en educación, se abordan a partir de las categorías identificadas por MORRIS y COGAN (2009), quienes indagan acerca de la operacionalización de la educación cívica en el currículum escolar.

Posteriormente se explica en qué consistió la reforma de la educación media superior en México y el contexto político en el que se suscitó, para analizar el discurso pedagógico oficial en relación con la ciudadanía. Con dicha base y el análisis curricular de dos asignaturas ('Introducción a las Ciencias Sociales' y 'Ética y Valores'), así como la reflexión sobre la propia práctica docente, se arrojan algunos resultados sobre las interpretaciones de la educación para la ciudadanía global en el bachillerato general mexicano, para analizar la influencia de la globalización en el cambio del modelo tradicional de la educación para la ciudadanía.

\section{LA CIUDADANÍA GLOBAL EN LAS POLÍTICAS EDUCATIVAS}

La ciudadanía global es un concepto que ha cobrado cada vez mayor centralidad en las políticas educativas. Especialmente a nivel de discurso, en el campo internacional. No obstante, se trata de un concepto poco claro, sujeto a múltiples interpretaciones. 
La cuestión que destaca es la amplitud de la noción de 'ciudadanía global'; es decir, la dificultad de hacerla operativa en educación. Esto se debe, fundamentalmente, a dos motivos. El primero, que es un concepto poco claro en el que la propia noción resulta una metáfora; una contradicción de términos e incluso un oxímoron (DAVIES y GRAHAM, 2009: 62). En segundo lugar, al ser aplicada a la educación, dicha noción implica un cierto grado de confusión.

En relación con el significado, la noción de 'ciudadanía global' posiblemente implica, tanto desde el punto de vista jurídico, como de la identidad colectiva, el sentido de pertenencia y el compromiso cívico (TAWIL, 2013: 2). En este sentido, se desprenden algunas preguntas ¿es la educación para la ciudadanía global una expresión del propósito fundamental de los sistemas educativos?; ¿se refiere a un área concreta del proceso de enseñanza aprendizaje? En tal caso ¿cuáles son los límites de dicho dominio?; ¿cómo se relaciona con otras áreas de aprendizaje, a menudo superpuestas, asociadas con la socialización cívica y política?

Tradicionalmente, la noción de ciudadanía se ha relacionado con la pertenencia individual a una comunidad política contenida en los límites del Estado Nación. Ello comprende un sentido de pertenencia a una comunidad política nacional pero también una forma de acción, con implicaciones a nivel de responsabilidades y derechos.

En la concreción del concepto de ciudadanía a través de una carga de derechos y responsabilidades, suelen reconocerse dos tradiciones: la 'cívica republicana' y la liberal (PÉREZ-CASTRO, 2001). En la primera, la ciudadanía se caracteriza por hombres virtuosos y por un modelo justo de gobierno, que supone obligaciones y una 'virtud cívica'. En la perspectiva liberal, el Estado es garante de los derechos de los individuos.

LISTER (1998) sugiere dos aspectos de la ciudadanía: ser ciudadano y actuar como ciudadano:

"Ser ciudadano significa gozar de los derechos necesarios para la agencia, así como para la participación social y política. Actuar como ciudadano implica el cumplimiento de todo el potencial de su estatus" (LISTER, 1998: 328-329).

Desde la perspectiva de la diversidad, ISIN y WOOD (1999) sugieren que una concepción de ciudadanía necesita basarse en el ethos de pluralización como la respuesta necesaria a las tensiones de múltiples identidades y lealtades que de hecho existen en el mundo globalizado contemporáneo.

En cualquier caso, la noción de ciudadanía es controvertida, sujeto de variedad de interpretaciones, no solamente en sociedades divididas, también en el caso de las relaciones entre poblaciones indígenas y otras minorías culturales en el interior de un Estado. Por otra 
parte, los derechos asociados a la ciudadanía a menudo son negados a los grupos de migrantes, en particular a los refugiados.

Cualquier intento de incorporar la noción de ciudadanía más allá del Estado-Nación a nivel mundial, se vuelve aún más problemático, sobre todo desde una perspectiva jurídica. Aún con el anuncio que hacen las Naciones Unidas, acerca del surgimiento de una comunidad política mundial, sus miembros están compuestos de naciones, no de individuos. Así pues, pese a la forma en que la globalización está afectando a las concepciones tradicionales de la ciudadanía dentro de los límites del Estado-Nación, la noción de 'ciudadanía global' sigue siendo una metáfora; más aún, una contradicción ${ }^{1}$.

Sin embargo, la concepción tradicional de ciudadanía nacional está cambiando bajo la influencia de múltiples procesos asociados con la globalización. Se habla de la emergencia de formas post-nacionales de ciudadanía (SASSEN, 2002) vinculadas a la internacionalización del comercio y las finanzas, un mayor acceso a la información, el conocimiento y los valores difundidos en todo el mundo a través de los nuevos medios digitales, el aumento de la migración y la movilidad a través de las fronteras, la degradación del medio ambiente asociada al cambio climático global, así como la consolidación de los organismos internacionales de la gobernanza global. El aumento de la aceleración, la complejidad y la interdependencia de los múltiples procesos de cambio económico, tecnológico, ambiental, social y político, están contribuyendo a la expansión de las relaciones sociales a través del mundo.

Dichas concepciones 'post-nacionales' de ciudadanía son, en parte, vinculadas a las comunidades sociales y políticas transnacionales, el activismo civil y a las nuevas formas de identificación y movilización global. Sin embargo, a pesar de estas transformaciones, el Estado sigue siendo el referente para la ciudadanía.

Más allá del nivel jurídico, la ciudadanía puede desarrollarse como un sentido de pertenencia a una comunidad política global a través de la identificación con valores humanistas que inspiran principios como la igualdad de derechos, respeto por la dignidad humana, justicia social y solidaridad internacional, en la que se basa el ethos de los marcos normativos internacionales. En este sentido, se argumenta la pertinencia de referirse a una 'ciudadanía cosmopolita' (CORTINA, 1997) más que una ciudadanía global².

\footnotetext{
1 ¿De qué ciudadanía global hablamos, cuando hay personas que mueren ahogadas diariamente en el Mediterráneo, en la búsqueda desesperada de un lugar en el mundo para vivir, lejos del terror de la guerra? ¿A qué ciudadanía democrática hacemos referencia, mientras México registra -solamente en lo que va del sexenio de Peña Nieto- más muertes y desapariciones forzadas que las dictaduras latinoamericanas de finales de siglo $\mathrm{XX}$ ?

${ }^{2}$ El cosmopolitanismo tiene como fundamento el reconocimiento de la legitimidad del principio de universalidad. Dicho principio resulta clave desde la perspectiva humanista, humanitaria, de los derechos
} 
Para despejar, en algún grado, la confusión que genera dicha noción -que es, de por sí, compleja y polémica- al ser aplicada a la educación, SHULTZ (2007) propone una revisión del rol del ciudadano global, en el sentido de construir espacios de entendimiento y compromiso que se extiendan más allá de las fronteras tradicionales, así como crear nuevas formas de negociar las relaciones globales. Porque:

"si los ciudadanos de las naciones bien estantes aprenden que su rol como ciudadanos globales es competir en el mercado global, las estructuras que mantienen a los países de menor bienestar en la desigualdad y la marginación, serán perpetuadas, si no fortalecidas" (SHULTZ, 2007: 257).

Las polémicas definiciones de globalización y de ciudadanía global, reflejan tensiones sociales y discusiones más amplias. Por un lado, DOWER (2003: 7) sugiere que la ciudadanía global comprende tres componentes: "una afirmación normativa acerca de cómo debe actuar la humanidad; una afirmación existencial, acerca de cómo debe ser el mundo; y una afirmación aspiracional acerca del futuro".

Por otro lado, MCGREW (2000) presenta tres aproximaciones a la globalización que se utilizan como referente en este trabajo, para reflexionar acerca de la interpretación mexicana de ciudadanía global en educación- que reflejan diferentes posiciones en el sistema económico, político y social.

1) La aproximación neoliberal celebra el dominio de un mercado global único y los principios del comercio transnacional liberal. Desde esta perspectiva, el ciudadano global, es aquél que participa exitosamente en la economía liberal conducida por el capitalismo y la tecnología. En este caso, la posición privilegiada resulta de la capacidad emprendedora individual en el sector privado. Se asocia a las personas que viajan alrededor del mundo llevando con ellas una agenda de "desarrollo global", a las que se suman los millones de emprendedores de negocios globales que arrojan como resultado una concepción del ciudadano global como un viajero.

En el ámbito educativo, esto se identifica en los programas que alientan al alumnado a viajar internacionalmente y adquirir experiencia global; una experiencia típica del mundo "desarrollado". Con el objetivo de incrementar la movilidad transnacional de conocimiento y competencias, desde el ámbito educativo se pretende facilitar dicha participación a través de la construcción de relaciones basadas en una concepción cultural, así como de capacidades como la adquisición de diferentes lenguas.

Ese tipo de ciudadanos globales, dice SHULTZ (2007: 252) "asumen que su posición de privilegio es natural y un signo de éxito. Aunque ellos mismos puedan apoyar 
esfuerzos de intervención como donaciones caritativas para mitigar el sufrimiento de aquellos que no son tan exitosos”.

2) La aproximación radical, en contraste, concibe la globalización como un modo acelerado del imperialismo occidental que utiliza el poder económico para la dominación. Desde esta perspectiva, el ciudadano global debe comprender cómo este sistema crea pobreza y opresión entre la mayoría de la población mundial y, por lo tanto, tiene como responsabilidad desafiar al Estado y a las estructuras corporativas que incrementan la marginalización de los países del sur.

En esa línea, se desarrolla un sentido de solidaridad global o nacional como sub producto de la hegemonía de la globalización económica. Las estructuras que sirven para reforzar dicha hegemonía incluyen instituciones internacionales como el Fondo Monetario Internacional (FMI), el Banco Mundial (BM) y la Organización Mundial de Comercio (OMC), también conocidas como las instituciones del Bretton Wood. "La globalización contemporánea, en su visión radical, es aquella implicada en la intensificación de la pobreza global, la privatización, el conflicto y la violencia” (MCGREW, 2000: 351).

El reto para esta aproximación a la ciudadanía global radicalizada, consiste en construir solidaridad a través del quiebre de dichas estructuras globales de opresión. Desde esta lógica, se hace un llamado a la acción contra las instituciones globales, particularmente las instituciones financieras que son arquitectos del liberalismo económico global. El objetivo que persigue es el de crear un cambio radical en las relaciones norte - sur, a partir de la comprensión del lazo que existe entre las actividades económicas de dichas instituciones de opresión social y política, así como de destrucción económica.

En el ámbito educativo, el reto para esta aproximación, consiste en observar las relaciones globales más allá del maniqueísmo de buenos y malos.

3) La aproximación transformacional -que propone MCGREW (2000)-, concibe la globalización en sus dimensiones cultural, social, ambiental, política y económica, que arroja como resultados nuevos patrones de inclusión y exclusión, así como la erosión de las jerarquías norte - sur. Desde esta perspectiva, la globalización es concebida más que una nueva forma de imperialismo o una simple ruta hacia el mercado económico global, como la posibilidad de nuevas formas de gobernanza establecidas que incluyen intereses públicos y privados, agencias domésticas y transnacionales; es decir, un nuevo desarrollo de consenso emergido del post-Washington o el consenso de Ginebra.

Bajo la idea de que "otro mundo es posible", el ciudadano global pretende incluir y comprometer a otros en función de su humanidad común compartida. Así, la gente a través del mundo, se une para crear justicia social a través de una profunda compresión y 
acompañamiento, a través del cual se crean espacios democráticos para la construcción de comunidades inclusivas, y a través de la acción que enlaza la experiencia local con la experiencia global compartida. Se trata de una iniciativa política de construcción de capacidades centrada en la construcción de conocimiento.

Desde esta aproximación, se otorga especial importancia al hecho de crear espacios democráticos para comunidades y construir coaliciones que atraviesan fronteras locales, nacionales y regionales. A través de dicho proceso la ciudadanía es capaz de enlazar la acción en el nivel local y global para construir auténticos retos hacia aquellas fuerzas que perpetúan la presión, la pobreza y la marginalización, creando nuevas formas de inclusión y solidaridad transnacional.

El desafío político de esta aproximación a la ciudadanía consiste en asegurar que la acción sólo se consigue de la complejidad y la complicidad de las relaciones y el compromiso como ciudadano de un mundo global. En esta línea, se ha identificado el trabajo desarrollado en el Estado español por Intermón Oxfam (DE PAZ, 2007), en la línea del desarrollo de un modelo pedagógico de referencia para la Educación para la Ciudadanía Global, que cree en el poder transformador de la educación, y en ese sentido no solamente plantea unos medios didácticos, sino que plantea unos objetivos políticos del proceso educativo-socializador frente a los retos que plantea construir un modelo de sociedad en el contexto actual.

Las diversas concepciones de globalización sugieren un rol particular y una serie de acciones para un ciudadano global. Lo mismo sucede con la educación para la ciudadanía.

MOLLER (2002: 10) identifica al neoliberalismo como una filosofía particularmente estrecha para definir el rol del ciudadano: "para los neoliberales, la opción del consumo es garante de la democracia [...]; en ese sentido, la democracia se convierte, de un concepto político, a uno económico donde la equidad se confunde con la capacidad de consumir".

Los movimientos conocidos como 'globalifóbicos' que se afirman contra el neoliberalismo, conciben la ciudadanía activa como una fuerza radical necesaria para desafiar la hegemonía del mercado y para proteger el bienestar social y ambiental (APPLE, 2000; OSLER, 2000).

Queda claro que los múltiples procesos de globalización, tanto económicos como tecnológicos, ambientales o políticos, han ido transformando progresivamente las concepciones y prácticas tradicionales alrededor de la ciudadanía. Sin embargo, a pesar de dichas transformaciones, la base jurídica para la definición de la ciudadanía, así como su práctica, sigue estando situada en relación con el Estado-Nación. 
Las tensiones relacionadas con esta realidad cambiante explican, al menos en parte, la posible confusión y la resistencia potencial que puede encontrar una noción como la de 'ciudadanía global' que se ha introducido en el discurso internacional de las Naciones Unidas, en relación con la 'educación para el desarrollo'.

Se sobreentiende que el concepto de 'ciudadanía global', en dicho marco, se refiere a los intentos de introducir cuestiones de interés mundial y los elementos de una cultura cívica global emergente, en los programas de educación formal o no formal existentes (TAWIL, 2013:6); por lo tanto, el discurso de la educación para la ciudadanía global, pretende fomentar la adaptación y el enriquecimiento de los programas de educación para la ciudadanía local y nacional, cualquiera que sea su enfoque, con el contexto de la globalización intensificada.

\subsection{Interpretaciones de ciudadanía en educación}

Históricamente se ha asignado a la educación, una responsabilidad relativa a la formación de la ciudadanía y del fortalecimiento de la cohesión social. Una cuestión muy presente en el discurso pedagógico oficial del Estado Mexicano. Así pues, se asigna al sistema educativo un rol en la promoción de los conocimientos, competencias y valores que desarrollen un sentido de 'destino compartido' a través de la identificación individual con los ambientes social, cultural y político de una sociedad. Asimismo, se asigna a la escuela un papel en el fomento a la participación en la acción cívica y social, basada en un sentido de la responsabilidad individual hacia las comunidades.

Tal como plantea SHULTZ (2007: 257) "las ciudadanas y los ciudadanos globales aprenden que el mundo está determinado por estructuras que impiden un cambio y unas relaciones auténticas para el desarrollo". En este sentido, se ha desarrollado una nueva aproximación a la ciudadanía global a partir de la comprensión acerca de cómo las experiencias comunes de pobreza y marginalización se extienden más allá de las fronteras estatales. Sin embargo, esta es una reflexión que no ha permeado el currículum mexicano, ni en la educación básica ni en el nivel medio superior y solamente podría identificarse dicha reflexión crítica a nivel del currículum oculto, en vinculación directa con el papel del docente.

En el entendido de que compartimos un planeta y un sentido de humanidad, las ciudadanas y los ciudadanos globales aprenden, según esta última autora, que la compasión y el cuidado se convierten en conexiones poderosas que atraviesan las típicas fronteras del Estado, la nacionalidad, la raza, la clase o el sexo. Las relaciones de poder se negocian, a partir del diálogo y la deliberación, en contextos localizados como espacios de interacción. 
Decir que en México este es el aprendizaje que se está promoviendo, distaría profundamente de la realidad, marcada por la violencia en todos los niveles. Precisamente la falta de un diálogo respetuoso y razonado, así como la deliberación consciente, es lo que caracteriza los procesos políticos en México, que la mayoría de personas jóvenes pudieran adoptar como referente.

En ese sentido, los desafíos docentes incluyen metas de ciudadanía global, bajo el reconocimiento de que dicha ciudadanía necesita comprometerse en cuestiones y acciones más allá de su contexto local. La manera en la que se asume dicho compromiso, determina el tipo de ciudadanía global que se promueve en el proceso educativo.

La función social, cívica y política que se arroga a la educación, suele explicitarse en el currículum e implicar el diseño de una formación ciudadana ('cívica y ética', en la interpretación mexicana), que no necesariamente es explícita en la disciplina académica, pero, a menudo, implica un área amplia de la enseñanza aprendizaje. Un área que suele sobreponerse en diferentes grados, con materias como la Geografía, la Historia, las Ciencias Sociales, la Religión o los Estudios Ambientales. Asimismo, la educación ciudadana incluye los niveles de educación formal y no formal, y suele ser considerada como una parte esencial de la formación de la ciudadanía en cada contexto social.

La naturaleza controvertida de la educación para la ciudadanía puede conducir, pues, a una serie de interpretaciones y aproximaciones. Éstas, pueden ser minimalistas y conservadoras, en la línea de reproducir el orden social existente, o bien, más ambiciosas y críticas, dirigidas a la adaptación al cambio o, aún más, a la transformación de las dinámicas sociales existentes (LE MÉTAIS, 1997; KENNEDY, 1997; KERR, 1999).

A partir de un estudio comparativo reciente, en relación con la educación cívica, en seis sociedades (Australia, Hong Kong, Japón, Taiwán, Tailandia y los Estados Unidos de América), MORRIS y COGAN (2001) ofrecen una serie de categorías para analizar la operacionalización de la educación cívica en el currículum escolar a partir de la siguiente cuestión ¿cuál es la naturaleza de las políticas de Estado destinadas a promover el aprendizaje de valores y conceptos cívicos en las escuelas?

Para ofrecer una aproximación al caso mexicano, se indaga acerca de la interpretación que se hace del concepto de ciudadanía en el currículum de bachillerato general con enfoque propedéutico.

La tesis de MORRIS y COGAN (2001) es que el marco estructural básico en el que operan profesores y alumnos, tiene una relación directa con el medio a través del cual se operacionaliza la educación cívica en el currículo escolar. Dicho medio varía 
sustancialmente entre las sociedades en función del papel que juega el Estado en la definición del mismo.

A partir de los resultados obtenidos en su análisis ${ }^{3}$, estos autores plantean que las sociedades con Estados centralizados (como son Japón, Tailandia, Taiwán y Hong Kong) operacionalizan la educación cívica colocando el énfasis en la identidad nacional, el comportamiento moral, la religión y atributos personales como honestidad y civilidad. En cambio, en sociedades como Australia y Estados Unidos, el acento se coloca en aquellas áreas relacionadas con la democracia, los procesos políticos, los derechos humanos y la economía de libre mercado.

Frente a dicho planteamiento, debería suponerse que la educación cívica en México se operacionaliza a partir del comportamiento moral del alumnado, así como el refuerzo de la identidad nacional, dado el nivel de centralización que caracteriza al Estado mexicano. Sin duda existen dichos componentes; sin embargo, se verá que las dimensiones política y social de la ciudadanía -las áreas relacionadas con la democracia, los procesos políticos y los derechos humanos- tienen un papel central en el currículum. Lo que toca a la religión, depende del contexto escolar del que se trate ${ }^{4}$.

MORRIS y COGAN (2001) distinguen el grado en que las intenciones curriculares subrayan la educación acerca de, a través de (vía participación) o para (vía compromiso activo) la ciudadanía. Lo hacen a través de la revisión del currículum formal y el currículum oculto, sin reducirlo a la comparación descriptiva, sino elaborando distinciones analíticas útiles en relación a cuán explícito es el valor que se enseña o en el que se educa (LE MÉTAIS, 1997) ${ }^{5}$. Las interpretaciones que los diferentes currículums nacionales hacen del concepto de ciudadanía se plantean en términos de mínimos y máximos, a partir del contraste de indicadores, como se muestra en la siguiente Tabla 1.

\footnotetext{
${ }^{3}$ En cada país se analizaron dos a tres escuelas, distinguiendo pública, privada y, en algún caso, escuelas experimentales (demonstration school).

${ }^{4}$ El sistema educativo mexicano está regido por la Secretaría de Educación Pública (SEP) -equivalente al Ministerio de Educación en el Estado español- con base en el artículo tercero de la Constitución Política de los Estados Unidos Mexicanos y la Ley General de Educación (LGE, 1993). Dicho artículo constitucional sostiene que todo individuo tiene derecho a recibir educación pública, laica y gratuita. No obstante, el sector privado tiene un papel preponderante en la oferta de servicios educativos y, en dicho marco, la educación religiosa tiene gran influencia en México.

${ }^{5}$ Estos autores recomiendan revisar los análisis de LE MÉTAIS (1997) y KERR (1999) para profundizar en la forma en que estas sociedades expresan los valores que se espera que las escuelas enseñen, distinguiendo el grado de detalle o claridad con la que los valores se expresan. También sugieren una lectura de KENNEDY (1997) quien plantea que, cuanto más altas son las expectativas de la educación cívica en un país habrá menos probabilidades de que se refleje en la práctica significativa.
} 
Tabla 1. Interpretaciones de ciudadanía ${ }^{6}$

\begin{tabular}{|l|l|}
\hline \multicolumn{1}{|c|}{ Interpretaciones mínimas } & \multicolumn{1}{c|}{ Interpretaciones máximas } \\
\hline Educación cívica & Educación para la ciudadanía \\
\hline Código agregado & Código integrado \\
\hline Enfocada en los contenidos & Enfocada en los procesos \\
\hline Basada en conocimientos & Basada en procedimientos \\
\hline Transmisión didáctica & Interpretación interactiva \\
\hline Basada en los textos & Variedad de recursos \\
\hline Fácil de asimilar y medir en la práctica & Difícil de asimilar y medir en la práctica \\
\hline Formal & Participativa \\
\hline Presencia sutil en el proceso educativo & Presencia robusta en el proceso educativo \\
\hline Enfoque exclusivo & Enfoque inclusivo \\
\hline Elitista & Activista \\
\hline
\end{tabular}

A partir de dichos elementos, se analiza la interpretación que se hace de la educación para la ciudadanía en el currículum del bachillerato general mexicano con enfoque propedéutico.

\subsection{Metodología de análisis de las categorías de ciudadanía en educación}

Para analizar cada una de las dimensiones señaladas se adoptó la siguiente metodología. Primero, ser realizó una lectura crítica de la reforma integral a la educación media superior (RIEMS) identificando los elementos del discurso internacional acerca de la educación para la ciudadanía global que se reproducen y aquellos que se recontextualizan. Asimismo, se analizó el documento oficial del Plan Sectorial de Educación 2013-2018 para analizar la evolución del discurso. Después, se revisó el marco curricular común del nivel medio superior para identificar las competencias genéricas que tienen relación con la noción de 'ciudadanía global'.

Con base en el análisis del currículum del bachillerato general con enfoque propedéutico, se identificaron las categorías de la educación para la ciudadanía global en el área histórico social, a partir de la revisión de ocho libros de texto de las asignaturas de 'Ciencias Sociales', así como 18 libros de texto de 'Ética y Valores', que se cursan en el primer año del bachillerato. A ello se suma el trabajo etnográfico en un centro escolar privado de la provincia de Nayarit, durante el curso escolar 2014 - 2015.

El objetivo planteado consiste en analizar la manera en que los principios dominantes en el campo internacional son recontextualizados en el campo pedagógico, con la intención de identificar las contradicciones que se generan a partir de la brecha entre el discurso oficial y la práctica educativa.

\footnotetext{
${ }^{6}$ La traducción / interpretación de los conceptos utilizados por MORRIS y COGAN (2001) es propia.
} 
De acuerdo con la propuesta de BERNSTEIN (1990), el análisis del dispositivo pedagógico debe hacerse a través de la revisión del conocimiento educativo: su contenido, la forma de transmitirlo y la forma de evaluarlo. De esa manera, se hace posible identificar las relaciones de poder, externas a la educación, que se transmiten en la escuela. La expresión de dichas relaciones de poder estará presente en el currículum, en la pedagogía y en la evaluación.

En este trabajo se analiza la dimensión curricular, en relación con el discurso de la educación para la ciudadanía global como dispositivo pedagógico, aunque se tienen en cuenta elementos de las relaciones pedagógicas (especialmente en el currículum oculto) identificadas a partir de la observación participante en un centro educativo de bachillerato ${ }^{7}$.

Para ello, se identifican los temas del programa del área histórico social de bachillerato en los que se hace referencia a cuestiones vinculadas con la ciudadanía, en general, y con la ciudadanía global, en su caso.

En años recientes, se han suscitado un sinfín de reformas curriculares en los distintos subsistemas de la educación media superior (EMS) en México. En el análisis de la reforma integral a la educación media superior (RIEMS) de 2009, se puso de manifiesto la conflictividad que implicó la definición de un marco curricular común (MCC) para el bachillerato.

Definir cuáles son los conocimientos, las habilidades y actitudes que todo bachiller debe poseer al finalizar sus estudios no es un asunto de consenso. Por ello, en este trabajo se toma como referencia el perfil básico del egresado de bachillerato que recoge la normatividad vigente. Dicho perfil hace referencia a los desempeños comunes que los egresados del bachillerato deben conseguir independientemente de la modalidad y subsistema que cursen.

En dicho marco se adopta el sistema de formación por competencias en las que se definen las genéricas y las disciplinares básicas, que son comunes a todas las personas egresadas del sistema de EMS; las disciplinares extendidas, que son de carácter propedéutico y las competencias profesionales, que no se abordan en este trabajo.

En el área de Humanidades y Ciencias Sociales, disciplinas como Filosofía, Ética y Lógica se han considerado "de carácter transversal” según el documento de Reforma que se toma como base (SEMS, 2008:71).

\footnotetext{
${ }^{7}$ Además de BERNSTEIN (1990), diversos autores de la denominada 'nueva sociología de la educación' han centrado sus investigaciones en el currículum como expresión de las relaciones de poder que subyacen a la selección y organización del conocimiento educativo (APPLE, 1989; YOUNG y GAMBLE, 2006; MULLER, 2000).
} 
El caso de la asignatura de 'Ética y Valores' es peculiar, ya que resulta una suerte de continuidad con la 'Formación cívica y ética' que forma parte del currículum de la educación básica, desde el primer año de primaria hasta el último de secundaria (6 a 15 años de edad). En el caso del bachillerato general con enfoque propedéutico, la frecuencia de dicha asignatura suele ser de tres clases por semana durante los dos primeros semestres.

Es de suponerse que los conocimientos vinculados con el concepto de ciudadanía global estarán contenidos en el área histórico - social. De dicho supuesto se desprende que, en el marco curricular común del bachillerato mexicano, no hay lugar para dicha propuesta, a menos que se abordase, efectivamente, desde una perspectiva transversal.

\section{LA REFORMA INTEGRAL DE LA EDUCACIÓN MEDIA SUPERIOR EN $\underline{\text { MÉXICO }}$}

Una cuestión clave en la RIEMS es la obligatoriedad de la educación media superior, que contempla una escolarización hasta la mayoría de edad (18 años). Se trata, pues, de un nivel educativo del que egresan ciudadanas y ciudadanos, nuevos votantes.

En relación con la educación para la ciudadanía, el documento oficial de la Subsecretaría de la Educación Media Superior (SEMS) de la Secretaría de Educación Pública (SEP), sugiere que "no se debe perder de vista que de la EMS egresan individuos en edad de ejercer sus derechos y obligaciones como ciudadanos" y que, como tales, "deben reunir, en adición a los conocimientos y habilidades que definirán su desarrollo personal, una serie de actitudes y valores que tengan un impacto positivo en su comunidad y en el país en su conjunto" (SEMS, 2008: 4).

\subsection{Contexto político en que se suscita la reforma}

En el año 2000, Vicente Fox Quesada ganaba las elecciones presidenciales en representación de la denominada "alianza por el cambio" del derechista Partido Acción Nacional (PAN) y su socio coyuntural, el Partido Verde Ecologista de México (PVEM) ${ }^{8}$. En ese mismo año se empezó a implementar el Programa Integral de Formación Cívica y Ética (PIFCYE) de la SEP para la educación básica (primaria y secundaria) ${ }^{9}$.

Las elecciones de 2000 representaron un parteaguas en la historia de México debido a que, por primera vez en la historia moderna del país, el Partido Revolucionario

\footnotetext{
${ }^{8}$ En las elecciones federales del año 2006, el PVEM se alió con el PRI, postulando a Roberto Madrazo Pintado para la presidencia.

${ }^{9}$ Levinson (2004) analiza los retos que enfrenta la reforma al programa para la educación cívica de secundaria en México, a partir de los actores involucrados en su implementación. Su tesis es que el nuevo planteamiento en materia cívica en México asume como base una pedagogía democrática en la ausencia de una cultura política democrática, por lo que dicha reforma se recibe con gran escepticismo.
} 
Institucional (PRI) resultó derrotado en una elección presidencial. Desde su fundación en 1929 -con el nombre de Partido Nacional Revolucionario (PNR)-, todos los presidentes mexicanos habían sido los candidatos del PRI ${ }^{10}$.

Desde el año 2000 y hasta el 2012, en que el PRI recupera la presidencia con Enrique Peña Nieto -actual mandatario hasta el final del sexenio en curso, en 2018-, gobernó en México el Partido Acción Nacional. En las elecciones federales del año 2006, los resultados dieron al candidato presidencial del PAN, Enrique Calderón Hinojosa, un estrecho margen (0,56\%) ante el candidato del Partido de la Revolución Democrática (PRD), Andrés Manuel López Obrador (hasta entonces jefe de gobierno de la capital mexicana y actual presidente del Movimiento de Renovación Nacional, (MORENA). López Obrador no reconoció el resultado, alegando fraude y denunciando las irregularidades que se presentaron en la jornada electoral; un hecho en sintonía con la crisis política que atravesaba el país desde tiempo atrás y que colocó a Calderón Hinojosa en una crisis de legitimidad durante todo su mandato.

La implementación de la reforma de la educación secundaria en México, que dio pie a la creación del PIFCyE, correspondió al gobierno de Fox Quesada y estuvo marcada por un periodo de transición política que fue de una "larga historia de gobiernos autoritarios corruptos" (LEVINSON, 2004: 1), a lo que prometía ser un régimen democrático.

Las múltiples reformas en materia educativa se gestaron en dicho mandato y se concretaron durante el período de gobierno de Calderón Hinojosa marcado, a su vez, por la denominada "guerra contra el narcotráfico"11.

La reforma política que Calderón propuso al inicio de su gobierno se promulgó en agosto del 2012, al final del sexenio. En materia de educación destacan los resultados de las

\footnotetext{
${ }^{10}$ En 1990, en un debate televisivo dirigido por Enrique Krauze (discípulo del premio Nobel de literatura e intelectual orgánico de Estado, Octavio Paz), el escritor peruano Mario Vargas Llosa -también premio Nobel de literatura- calificó a México de "dictadura perfecta", en referencia al gobierno del PRI: "tiene las características de la dictadura: la permanencia, no de un hombre, pero sí de un partido. Y de un partido que es inamovible [...] es una dictadura sui géneris, que muchos otros en América Latina han tratado de emular [...]; tan es dictadura la mexicana, que todas las dictaduras latinoamericanas desde que yo tengo uso de razón han tratado de crear algo equivalente al PRI". Vargas Llosa hablaba en un evento patrocinado por Televisa, consorcio mediático que, sin pelos en la lengua, se define como "soldado del PRI" (El País, 1 de septiembre de 1990).

${ }^{11}$ La guerra contra el narcotráfico inició como un conflicto armado interno que enfrentaba al Estado mexicano y los Grupos de Autodefensa Popular y Comunitaria contra los cárteles que controlan diversas actividades ilegales, principalmente el tráfico de drogas. Esta situación comenzó el 11 de diciembre de 2006, cuando el gobierno federal de Calderón anunció un operativo contra el crimen organizado en el estado de Michoacán (Operación Conjunta Michoacán), donde a lo largo de 2006 se habían contabilizado cerca de 500 asesinatos de miembros de los cárteles del narcotráfico. Como resultado de esa declaración de guerra, a la fecha, el saldo es de 186 mil personas asesinadas, 29 mil desaparecidas y 200 mil desplazadas, además de incalculables violaciones a los derechos humanos y una caída en picada de la confianza ciudadana en las instituciones encargadas de garantizar el estado de derecho (Taula per Mèxic, 2016).
} 
pruebas aplicadas por la OCDE en dicho período, en que el alumnado mexicano nunca pudo llegar al promedio de los otros países miembros. Además, la escasa cobertura educativa generó que millones de jóvenes quedaran fuera del bachillerato.

La Reforma Integral a la Educación Media Superior (RIEMS, 2009) responde al proyecto educativo del gobierno de Calderón. No obstante, es preciso tener en cuenta que, en México, en las últimas cuatro décadas, las reformas en materia educativa han sido incesantes.

En la actualidad, la reforma educativa de 2012-2013 presentada por el presidente de la República, Enrique Peña Nieto, dentro del marco de los acuerdos y compromisos establecidos en el Pacto por México, sigue siendo motivo de enorme crispación política ${ }^{12}$. Se puede afirmar que México está sumido en una auténtica crisis de gobernabilidad a partir de las movilizaciones de un importante sector del magisterio que rechaza de tajo la última reforma educativa.

\subsection{La aproximación a la ciudadanía global en la reforma}

Hasta el año 2007, en que se gesta la Reforma Integral a la Educación Media Superior (RIEMS), existían en México una serie de subsistemas que operaban de manera independiente, sin correspondencia a un panorama general articulado y sin que existiera suficiente comunicación entre ellos.

El reto que se propone la RIEMS ${ }^{13}$ consiste en encontrar objetivos comunes a dichos subsistemas para crear un Sistema Nacional de Bachillerato (SNB) con base en cuatro pilares: construcción de un Marco Curricular Común (MCC), definición y regulación de las modalidades de la oferta de la Educación Media Superior, profesionalización de los servicios educativos y Certificación Nacional Complementaria.

En el discurso que introduce la reforma, se asevera que la competitividad de México depende en buena medida del adecuado desarrollo de este nivel educativo. Por lo tanto, "la

\footnotetext{
${ }^{12}$ La última reforma educativa fue aprobada por la Cámara de Diputados el 20 de diciembre de 2012 y por el Senado de la República el 21 de diciembre del mismo año. En febrero de 2013, la reforma fue declarada constitucional por el Poder Legislativo Federal, promulgada por el Ejecutivo el 25 de febrero de 2013 y publicada al día siguiente en el Diario Oficial de la Federación. El 10 de septiembre de 2013, Peña Nieto promulgó la reforma a la Ley General de Educación, la Ley del Instituto Nacional para la Evaluación de la Educación y la Ley General del Servicio Profesional Docente; los tres decretos fueron publicados en el Diario Oficial al día siguiente.

${ }^{13}$ La RIEMS se llevó a cabo en el período que va de los años 2007 a 2009, en el gobierno panista de Calderón Hinojosa. El documento oficial de la Subsecretaría de la Educación Media Superior (SEMS) de la SEP es del año 2008 y recoge aportaciones de las autoridades educativas de los diferentes Estados de la República, de la Red de Bachilleratos de la Asociación Nacional de Universidades e Instituciones Educativas (ANUIES), del Consejo de Especialistas de la SEP, de la Universidad Nacional Autónoma de México (UNAM), del Instituto Politécnico Nacional (IPN) y de diversos especialistas en temas educativos.
} 
cobertura y la calidad en la EMS constituyen el supuesto fundamental para que el país pueda dar respuesta a los desafíos que presenta la economía globalizada en un marco de equidad" (SEMS, 2008: 4).

A partir del análisis del discurso oficial, queda claro que 'la calidad de la educación' es el eje que orienta la reforma integral a la EMS. En el análisis del currículum único y las competencias genéricas que constituyen el perfil del egresado de dicha etapa educativa, se identifican algunos elementos del discurso de la educación para la ciudadanía global como prioridad mundial.

Tal como está definido en el documento (SEMS, 2008:50) las competencias son la unidad común para establecer los mínimos requeridos para obtener el certificado de bachillerato sin que las instituciones renuncien a su particular forma de organización curricular.

En el documento oficial se explicita que la OCDE ha señalado que, hasta ahora, la evaluación de competencias realizada por el organismo a través del Programa Internacional para la Evaluación del Estudiante (PISA, Programme for International Student Assessment), se ha centrado exclusivamente en aspectos que evalúan la capacidad de responder a demandas complejas, utilizando y movilizando recursos psicosociales (incluyendo habilidades y actitudes) en un contexto particular. Plantea, sin embargo, que debe preverse la incorporación de otras categorías en evaluaciones futuras.

En el currículum de bachillerato se incluyen las competencias lingüísticas, esenciales para la comunicación humana; las habilidades sociales, de cuidado de sí mismos, y las competencias morales que permiten el desarrollo personal y la convivencia armónica; también se hace referencia a las habilidades de pensamiento de orden superior, a la resolución de problemas no sólo prácticos, también teóricos, científicos y filosóficos (SEMS, 2008:51).

El ex sub secretario de Educación Básica, Olac Fuentes Molinar, en una reciente entrevista realizada por un diario crítico de circulación nacional (La Jornada, lunes 8 de agosto de 2016), señala la desmesura de pretensiones que reflejan los productos de la reforma educativa en México. En el ciclo inmediato anterior al bachillerato, que es la secundaria (tres años, entre los 12 y los 15 de edad) - "el más retador y disfuncional de nuestros niveles escolares"-, al terminar el ciclo, lo que se espera del alumnado está totalmente alejado de la realidad ${ }^{14}$.

\footnotetext{
${ }^{14}$ El titular de la entrevista periodística que se cita dice "la SEP se olvida que tiene alumnos de carne y hueso", en relación con la reflexión del académico que asegura que "el país perderá otra oportunidad de
} 


\begin{abstract}
"El criterio de selección de temas no es el interés de los alumnos ni sus necesidades realmente básicas para mejorar su vida y entender ciertas cosas esenciales de la naturaleza y la sociedad, ni estimular su curiosidad y capacidad de aprender. [...] Los temas están seleccionados a partir de las exigencias implacables de las disciplinas; al parecer, cada grupo de especialistas consideró que la suya era la única importante y eso llevó al exceso en la cantidad de temas, como a la complejidad inmanejable de muchas de ellas" (FUENTES, 2016).
\end{abstract}

Una vez más -dice el ex subsecretario-, la autoridad educativa elaboró un currículum convencional que mantiene la estructura tradicional de la disciplina tal y como se ha enseñado (siempre) en la escuela, precedido de un discurso pedagógico "que colecciona posiciones progresistas y renovadoras" (ibid). Pretensiones que contrastan con los resultados de los estudiantes mexicanos en las pruebas PISA, donde éstos últimos son de un nivel menos que elemental: alrededor de la mitad de alumnos de educación básica es matemáticamente analfabeta; y en lectura, 40 por ciento no tiene más que comprensión literal elemental, esto es, lo que está expreso. Pero, además, subraya, el otro problema está en la desigualdad. Los pobres salen mucho peor que los menos pobres.

Una cuestión similar ocurre en el bachillerato, donde encontramos un discurso oficial que recupera los conceptos más innovadores en pedagogía, proponiendo el paso de una perspectiva conductista a una constructivista de la enseñanza "que elimina de las prácticas educativas la memorización no significativa, favorece el aprendizaje basado en resolución de problemas, que parte de su identificación y la aplicación de las herramientas necesarias para su resolución. Además, confiere un papel sumamente importante al desarrollo de capacidades de aprendizaje autónomo y se nutre fuertemente del trabajo colaborativo" (SEMS, 2008: 52). No obstante, la realidad pedagógica en el bachillerato, en general, sigue siendo la del modelo tradicional, de acuerdo con los resultados del trabajo etnográfico y los testimonios del alumnado.

\title{
3. INTERPRETACIONES DE LA EDUCACIÓN PARA LA CIUDADANÍA GLOBAL EN EL BACHILLERATO GENERAL MEXICANO
}

La concepción de la educación para la ciudadanía en México se identifica en la formación cívica y ética que forma parte del currículum de la educación básica, desde el primer año de primaria hasta el último de secundaria. En el caso del bachillerato general con enfoque propedéutico (de los quince a los dieciocho años), se identifican áreas de oportunidad para introducir el discurso de la ciudadanía global en algunas asignaturas y en las competencias que se esperan del bachiller egresado.

En relación con el modelo pedagógico que pudiera contemplar la educación para la ciudadanía global como prioridad, es interesante rescatar algunas de las competencias

empezar a salir de este panorama de catástrofe de logros de aprendizaje. Desearía que las autoridades recapacitaran y consideren la realidad de las escuelas". 
genéricas diseñadas para el bachiller, como son la capacidad de pensar crítica y reflexivamente y de sustentar una postura personal sobre temas relevantes, a nivel general; más específicamente, toca analizar la capacidad de participar con responsabilidad en la sociedad con una conciencia cívica y ética en la vida de su comunidad, región, México y el mundo; así como la capacidad de mantener una actitud respetuosa hacia la interculturalidad y la diversidad de creencias, valores, ideas y prácticas sociales.

En relación con las prioridades establecidas por el Programa de Educación de la UNESCO (2014-2017) y la iniciativa mundial del Secretario General de las Naciones Unidas "La educación ante todo", se identifica también la introducción del enfoque de educación para el desarrollo sostenible. En este sentido, queda claro que hay un nivel de reproducción del discurso oficial, al menos en el nivel formal, en el diseño del marco curricular común del bachillerato con enfoque de competencias.

De acuerdo con los atributos establecidos para dicha competencia (SEMS, 2008: 101), la persona egresada del bachillerato privilegia el diálogo como mecanismo para la solución de conflictos; toma decisiones a fin de contribuir a la equidad, bienestar y desarrollo democrático de la sociedad; conoce sus derechos y obligaciones como mexicano y miembro de distintas comunidades e instituciones, y reconoce el valor de la participación como herramienta para ejercerlos. Contribuye a alcanzar un equilibrio entre el interés y bienestar individual y el interés general de la sociedad. Actúa de manera propositiva frente a fenómenos de la sociedad y se mantiene informado; advierte que los fenómenos que se desarrollan en los ámbitos local, nacional e internacional ocurren dentro de un contexto global interdependiente.

En relación con el planteamiento teórico de la ciudadanía global, la interpretación es congruente con los principios dominantes. Es decir que, a nivel de discurso, el dispositivo pedagógico de reproducción funciona. Sin embargo -y recuperando la reflexión de FUENTES (2016)- la realidad de los estudiantes mexicanos poco tiene que ver con los máximos establecidos en las metas discursivas.

Tal como está formulada la reforma, la responsabilidad de promover la creación de ambientes de aprendizaje y situaciones educativas apropiadas al enfoque por competencias -favoreciendo las actividades de investigación, el trabajo colaborativo, la resolución de problemas, la elaboración de proyectos educativos interdisciplinares, entre otros- recae estrictamente en la persona del o la docente. Sucede lo mismo con la evaluación de las competencias de los estudiantes. Lo único que contempla el documento oficial es que este tipo de evaluación requiere el uso de métodos diversos, por lo que los docentes deberán contar con las herramientas para hacerlo. 
La responsabilidad de hacer efectivo el planteamiento pedagógico de la RIEMS recae en el aula. Por lo tanto, el cambio de paradigma en el nivel medio superior del sistema educativo mexicano dependerá, en gran medida, de las posibilidades y capacidades del profesorado. Aunque, desde otro punto de vista, también otorga a dicho colectivo la capacidad transformadora y estructurante.

Con base en la revisión de los libros de texto de las asignaturas de 'Ciencias Sociales' y 'Ética y Valores', así como la propia práctica docente en las asignaturas de 'Geografía Económica' y 'Ecología y Medio Ambiente', se identifican las áreas disciplinares en las que se introduce, aunque sea de manera incipiente, la noción de 'ciudadanía global'.

En lo que toca a la asignatura de 'Introducción a las Ciencias Sociales', existe la posibilidad de abordar las dimensiones civil e intercultural de la ciudadanía -en relación con las dimensiones propuestas por CORTINA (1997)- en el ámbito que sugiere que el alumnado 'identifique problemas sociales actuales de México y el mundo'. En lo que toca a las dimensiones de la ciudadanía política y social, el enfoque es estrictamente nacional, sin perspectiva global. La sugerencia, en este sentido, es que el alumnado 'analice la interacción del individuo con las instituciones del Estado mexicano'.

En relación con la asignatura de 'Ética y Valores', se promueve el respeto a los derechos humanos, así como la comprensión de 'la importancia del respeto a la identidad nacional ante los fenómenos asociados a la globalización'. En este sentido, se pone el acento en aquellos aspectos del país que deben preservarse ante la globalización, concebidos como 'una lucha contra las nuevas formas que adopta el colonialismo y el dominio cultural y hegemónico'. En consecuencia, se enfatiza la importancia de la recuperación histórica de las señas de identidad de los pueblos indígenas, así como el cultivo de los valores que se requieren para la convivencia y la soberanía.

Para profundizar el análisis, se revisa el enfoque por competencias en la formación cívica, ética y valoral, con base en la crítica pedagógica elaborada por FIERRO (2011), quien sugiere que los libros de texto contienen un enfoque del civismo cargado de información en un tono oficialista y anacrónico. La ética, por su parte, se aborda bajo un enfoque que regresa a los planteamientos del siglo XIX, centrados en la prescripción de 'lo correcto' desde la autoridad, alejándose del propósito de interpelar a la vida y animar la reflexión y la deliberación autónoma.

Dicho enfoque se mantiene en el bachillerato, aunque con una expresión formal de la orientación hacia las competencias. No obstante, el planteamiento sigue enfatizando una gran cantidad de contenidos a transmitir, inabarcables en los tiempos previstos para su desarrollo en el período lectivo. 


\section{CONCLUSIONES}

La ciudadanía global es un concepto que ha cobrado cada vez mayor centralidad en el discurso pedagógico internacional. No obstante, se trata de un concepto poco claro, sujeto a múltiples interpretaciones. Este trabajo aborda la interpretación de dicha noción en la educación ciudadana en México para identificar de qué manera, las tendencias educativas globales, han ido modificando el modelo tradicional de la educación para la ciudadanía.

El objetivo consiste en analizar la manera en que los principios dominantes en el campo internacional son recontextualizados en el campo pedagógico nacional. La tesis central es que existe una brecha entre el discurso pedagógico oficial -que reproduce principios progresistas- y la operacionalización de la educación para la ciudadanía, en un sistema educativo conservador. Esa brecha se intensifica con las incesantes reformas educativas que se han suscitado en los últimos años en México y obstaculiza la transformación del modelo tradicional de la educación para la ciudadanía, por el escepticismo con que es recibido por los actores; especialmente, el personal docente.

Para fundamentar dicho planteamiento, se analiza el discurso con el que se justifica la reforma integral de la educación media superior (RIEMS, 2009) y el enfoque por competencias; se hace una revisión curricular con base en los libros de texto del área histórico-social del bachillerato propedéutico y la reflexión se complementa con base en la propia práctica docente y el trabajo etnográfico en un centro educativo privado de la provincia de Nayarit durante el curso escolar 2014 - 2015 (PERAZA, 2015).

En México, la formación cívica y ética tiene lugar durante todo el período de educación básica (de los seis a los quince años) y se mantiene en el bachillerato propedéutico, especialmente en el área histórico social, a través de las asignaturas de 'Introducción a las Ciencias Sociales' y 'Ética y valores', aunque el planteamiento en la RIEMS para el marco curricular común, es la transversalidad.

Tal como se ha valorado desde el análisis pedagógico (FIERRO, 2011), el enfoque tradicional del civismo en México, está cargado de información en un tono oficialista y anacrónico. La ética, por su parte, se aborda bajo un enfoque que regresa a los planteamientos del siglo XIX, centrados en la prescripción de 'lo correcto' desde la autoridad, alejándose del propósito de interpelar a la vida y animar la reflexión y la deliberación autónoma.

En contraste, el discurso en la RIEMS recupera los conceptos más innovadores en pedagogía, proponiendo el paso de una perspectiva conductista a una constructivista de la enseñanza, confiriendo un papel sumamente importante al desarrollo de capacidades de aprendizaje autónomo que se nutre fuertemente del trabajo colaborativo. No obstante, la 
realidad pedagógica en el bachillerato, en general, sigue siendo la del modelo tradicional, de acuerdo con los resultados del trabajo etnográfico y los testimonios del alumnado.

Desde la perspectiva de la RIEMS, la responsabilidad de promover la creación de ambientes de aprendizaje y situaciones educativas apropiadas al enfoque por competencias recae estrictamente en la persona del o la docente. Sin embargo, como identifica LEVINSON (2004), el nuevo planteamiento en materia cívica en México asume como base una pedagogía democrática en la ausencia de una cultura política democrática, por lo que dicha reforma se recibe con gran escepticismo por parte del personal docente.

En lo que se refiere al planteamiento curricular, no se identifican los elementos que han ido transformando la concepción tradicional de ciudadanía, como podrían ser el activismo civil o las nuevas formas de identificación y movilización global. Tampoco se hace alusión al rol del ciudadano global, en lo que se refiere a la participación social y política, más allá de las fronteras nacionales.

De acuerdo con la literatura sobre EpCG, dicha noción implicaría un sentido de pertenencia y compromiso cívico a través de la identificación con valores humanistas que inspiran principios como la igualdad de derechos, respeto por la dignidad humana, justicia social y solidaridad internacional (CORTINA, 1997; DOWER, 2003; TAWIL, 2013). No obstante, las polémicas definiciones conceptuales, reflejan tensiones sociales y discusiones más amplias acerca de cómo debe actuar la humanidad, cómo debe ser el mundo y lo que se espera del futuro.

La distancia entre el discurso pedagógico progresista -más centrado en el deber sery la realidad política y social en México -caracterizada por la violencia en todos sus niveles y un tejido social desmembrado- no provee un marco de actuación congruente en el aula para un enfoque transformacional de la globalización, que implica negociar, a partir del diálogo y la deliberación, las relaciones de poder, en contextos localizados como espacios de interacción.

Desde la perspectiva de las áreas de oportunidad y los desafíos de la EpCG en México, es importante identificar el planteamiento del enfoque transformacional, que promueve una comprensión profunda de la justicia social, con la posibilidad de crear espacios democráticos para la construcción de comunidades inclusivas. En ese sentido, el enfoque pedagógico debería estar centrado en crear espacios democráticos con objetivos políticos claros en relación con el proceso educativo-socializador, frente a los retos que plantea construir un modelo de sociedad en el contexto actual.

La cuestión central que se pone de manifiesto en las interpretaciones de la ciudadanía en México, es un discurso instruccional cargado de buenas intenciones que no 
se traduce en prácticas pedagógicas. No obstante, los actores sociales que interactúan en el ámbito escolar, también son productores de discursos y prácticas, a partir de su capacidad recontextualizadora. Dicho principio deberá orientar la transformación del enfoque tradicional de la educación para la ciudadanía en México, tomando como referencia los principios gestados en el ámbito internacional hacia el enfoque de la educación para la ciudadanía global.

\section{REFERENCIAS}

APPLE, M. (1989): Maestros y textos (Madrid: Paidós).

APPLE, M. (2000): Between neoliberalism and neoconservatism: Education and conservation in a global context. En N.T. BURBULES C. (Ed.), Globalization and education: Critical perspectives, pp. 57-77 (Londres: Routledge).

BERNSTEIN, B. (1990): Clases, códigos y control. La estructura del discurso pedagógico (Vol. 4, Madrid: Morata).

CORTINA, A. (1997): Ciudadanos del mundo (Madrid: Alianza).

DAVIES, L., y GRAHAM, P. (2009): Global Citizenship Education, Challenges and Possibilities, en R. LEWIN, The Handbook of Practice and Research in Study Abroad; Higher education and the quest for global citizenship, pp. 61-79 (Nueva York: Rutledge).

DE PAZ, D. (2007): Escuelas y educación para la ciudadanía global, una mirada transformadora (España: Intermón Oxfam).

DOWER, N. (2003): An introduction to global citizenship (Edinburgh, UK: Edinburgh University Press).

FIERRO, M. (2011): Cívica y ética ¿asignatura elusiva o eludida? En COLMEX (El Colegio de México), Entre paradojas: a 50 años de los libros de texto gratuitos, pp. 469-498 (México: Colmex).

FUENTES, O. (2016): La SEP olvida que tiene alumnos de carne y hueso, p.7, lunes 8 de agosto (La Jornada, México).

GIDDENS, A. (1976): Las nuevas reglas del método sociológico, Primera edición en español de 1997 (Buenos Aires: Amorrortu).

ISIN, E.W.P., y WOOD (1999): Citizenship and identity (Londres: Sage).

KENNEDY, K. (1997): Citizenship education in review: Past perspectives and future needs. Citizenship education and the modern state, pp. 1-5.

KERR, D. (1999): Citizenship Education: an International Comparison (Qualifications and Curriculum Authority).

LE MÉTAIS, J. (1997): Values and aims in curriculum and assessment frameworks (International Review of Curriculum and Assessment Frameworks, Paper 1). School Curriculum and Assessment Authority, Londres. 
LEVINSON, B. A. (2004): Hopes and challenges for the new civic education in Mexico: toward a democratic citizen without adjectives, International Journal of Education Development, Elsevier, pp. 269-282.

LISTER, R. (1998): Citizen in action: Citizenship and community development in Northern Ireland context. Community Development Journal, Community Development Journal, 33(3), pp. 326-335.

MCGREW, A. (2000): Sustainable globalization? The global politics of development and exclusion in the new world order. En T. ALLEN y A. THOMAS (Eds.), Poverty and development into the $21^{\text {st }}$ century. pp. 345-364 (Milton Keynes, UK: Open University Press, y Oxford, UK: Oxfotd University Press).

MORRIS, P. y COGAN, J. (2001): A comparative overview: civic education across six societies, International Journal of Educational Research, pp. 109-123.

MOLLER, J. (2002): Democratic leadership in an age of managerial accountability. Documento presentado en la Conferencia CCEAM 2002: Exploring New Horizons in School Leadership for Democratic Schools (Universidad Umeå: Suecia).

MULLER, J. (2000): Reclaiming Knowledge: Social Theory, Curriculum and Education Policy (Londres: Rutledge Falmer).

PERAZA, C. (2015): Educación para la ciudadanía global en el bachillerato general mexicano. Un estudio de caso sobre la recontextualización del discurso oficial en el campo pedagógico. Tesis doctoral. Departamento de Sociología. Universidad Autónoma de Barcelona.

PÉREZ-CASTRO, E. (2011): Educación para una ciudadanía planetaria. XII Congreso internacional de teoría de la educación. Barcelona: Universitat de Barcelona. En línea.

SASSEN, S. (2002): Towards Post-national and Denationalized Citizenship. En E. ISIN y B. TURNER (Eds.), Handbook of Citizenship Education, pp. 277-291.

SEMS (SUBSECRETARÍA DE EDUCACIÓN MEDIA SUPERIOR) (2008): Reforma Integral de la Educación Media Superior en México: La Creación de un Sistema Nacional de Bachillerato en un marco de diversidad. México: SEP. (http://sems.gob.mx/es/sems/sistema_nacional_bachillerato) Obtenido de Secretaría de educación pública el 18 de junio de 2015.

TAULA PER MÈXIC (2016): Muerte, inseguridad e impunidad. A 10 años de la guerra contra el narcotráfico en México. https://taulapermexic.org/comunicados/decadaguerra/

TAWIL, S. (2013): Education for global citizenship: A framework for discussion, Colección ERF Working Papers (París: UNESCO), Num. 7, pp. 1 - 19.

YOUNG, M. y GAMBLE, J. (2006): Knowledge, Curriculum and Qualifications for South African Further Education (Pretoria South Africa: HSRC Press). 


\section{PROFESIOGRAFÍA}

\section{Cecilia Peraza Sanginés}

Doctora en sociología por la Universidad Autónoma de Barcelona; actualmente es profesora de tiempo completo, adscrita al Centro de Estudios Sociológicos, en la Facultad de Ciencias Políticas y Sociales de la Universidad Nacional Autónoma de México, donde realizó sus estudios de licenciatura. Sus principales temas de investigación comprenden la educación para la ciudadanía global; la educación en valores; la política educativa y el cambio en las instituciones de educación superior. Actualmente trabaja en una investigación que pretende comparar los efectos de la reforma educativa global hacia el modelo de la nueva gerencia pública en distintos países. Datos de contacto: Facultad de Ciencias Políticas y Sociales, Universidad Nacional Autónoma de México. E-mail: cecilia.peraza@politicas.unam.mx

Fecha de recepción: 15 de agosto de 2016.

Fecha de aceptación: 31 de diciembre de 2016. 